\title{
Posterior interosseous nerve entrapment
}

\section{by intramuscular lipoma arising from the}

\section{supinator muscle - a case report}

\author{
Omar Refai*, Mohamed Eslam Elsherif**, Ahmed A. Khalifa*** \\ *Orthopaedic Department, Assiut University Hospital, Assiut, Egypt \\ **Orthopaedic Department, Student's Hospital, Assiut University, Assiut, Egypt \\ $* * *$ Orthopaedic Department, Qena Faculty of Medicine and University Hospital, South Valley University, Qena, Egypt
}

Correspondence to: Ahmed A. Khalifa, MD, FRCS, MSc,

Assistant Professor and Consultant of Orthopaedic and Traumatology,

Qena Faculty of Medicine and University Hospital, South Valley University, Qena,

Kilo 6 Qena-Safaga Highway, Code 83523, Qena, Egypt,

Mobile phone: +201 224466 151, Phone: +20 965337 573, Fax.: + 20965337571,

E-mail: ahmed_adelo391@med.svu.edu.eg, ORCID: https://orcid.org/oooo-0002-0710-6487,

Web: www.svu.edu.eg

\begin{abstract}
Background: Lipomas are benign, slow-growing tumors frequently subcutaneous and asymptomatic, intramuscular lipoma constituting a rare subtype. However, a lipoma occurring nearby the proximal radius may cause posterior interosseous nerve (PIN) entrapment.

Case presentation: We described an uncommon case of a 45-year-old-man with a history of progressive, painless proximal right (dominant) forearm swelling for 4 months associated with PIN entrapment syndrome, presenting as fingers extension weakness. Intramuscular lipoma was observed in the supinator muscle in the magnetic resonance imaging (MRI). Lipoma surgical excision and release of the PIN through proximal forearm direct anterior approach was performed.

Results: The histopathological examination confirmed the diagnosis of benign intramuscular lipoma. The follow-up of the patient showed full recovery within three months postoperatively.

Discussion: Intramuscular lipoma is rare; however, it could originate from supinator muscle in the forearm, presenting with vague pain and could lead to compression of nearby neurovascular structures such as the PIN. Clinical evaluation and imaging studies, especially MRI, are crucial for diagnosis. If neural compression is evident, surgical resection is mandatory.

Conclusion: Intramuscular lipoma entrapping PIN is rare and requires early clinical diagnosis confirmed by imaging and neurophysiological studies, surgical excision being the method of choice for optimum functional outcomes.

Keywords: posterior interosseous nerve, entrapment, supinator muscle, intramuscular lipoma
\end{abstract}

\section{Introduction}

The posterior interosseous nerve (PIN) is one of the two major branches or the radial nerve, as it divides at the level of the lateral epicondyle. It represents the continuation of the deep motor branch of the radial nerve 
after it winds around the radius neck and enters the arcade of Frohse to pierce the supinator muscle $[1,2]$.

Various conditions can cause the PIN entrapment, such as fractures or dislocations around the elbow, neoplasms (lipoma, schwannoma), ganglion cysts, the fibrous arcade of Frohse, and vascular aneurysms [1-5].

Lipomas are common soft tissue mesenchymal tumors; typically, they are located subcutaneously, consisting of mature fatty tissue [6]. They are rarely located deep to the fascia, being further divided according to their location into intermuscular, intramuscular, interosseous, and parosteal lipomas $[6,7]$.

The intramuscular lipoma is a relatively uncommon lesion that accounts for about $1.8 \%$ of adipose tissue primary tumors and less than $1 \%$ of all lipomas $[6,7]$. Usually, it is asymptomatic, unless it is huge and causing disfigurement or compressing neurovascular structures [6].

We hereby present a case of PIN entrapment by an intramuscular lipoma originating within the supinator muscle in a male patient.

\section{Case presentation}

\section{History}

A 45-year-old male patient presented with insidious onset of weak extension of the middle and ring finger of his dominant right hand for 2 months. The patient noted swelling in his right upper forearm four months before his presentation, which was accompanied by attacks of dull aching pain, the patient reporting an increase of pain after manual working. No history of trauma or chronic illness was reported.

\section{Clinical examination}

We started by examining the neck and the shoulder, which were both normal; local examination of the forearm revealed a firm, immobile swelling about $5 \times 3 \mathrm{~cm}$ over the anterolateral aspect of the forearm just below the front elbow crease (the region of the mobile wad muscle), not pulsating, with no signs of inflammation of the overlying skin. He had a full painless elbow range of motion. Wrist and hand examination revealed intact wrist and finger flexion range of motion with full power. Conversely, weakness of right ring and middle finger extension was noted (graded as $3 / 5$ according to MRC scale) (Fig. 1). There was no sensory deficit in the superficial radial nerve distribution and the vascular examination was normal.

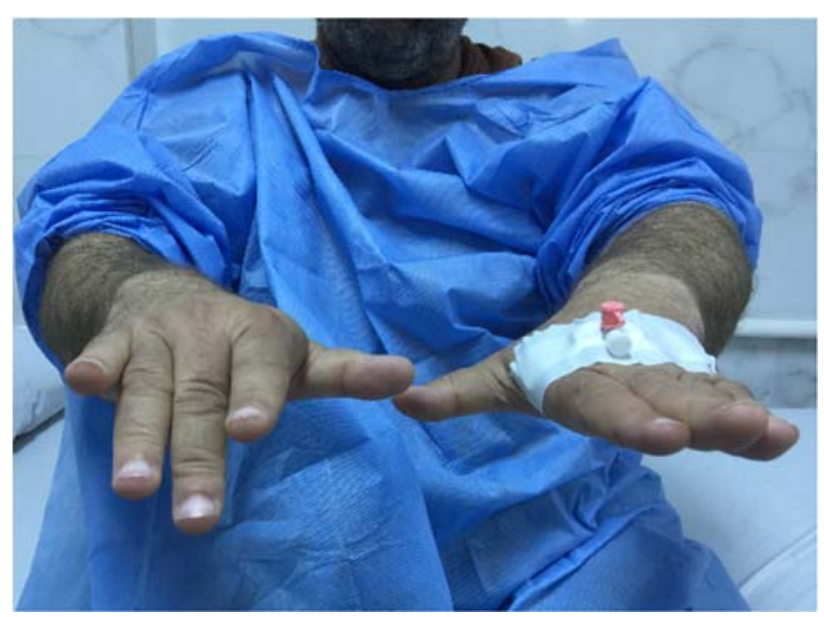

Fig. 1 Clinical examination of the patient revealing weakness of the right middle and ring fingers extension

\section{Imaging studies}

Plain radiographs of the elbow and proximal forearm were unremarkable (Fig. 2). However, magnetic resonance imaging (MRI) revealed evidence of fat signal lesion within the supinator muscle with intact bony structures, having a size of $3 \times 3.5 \times 2 \mathrm{~cm}$ in its maximum dimension. The lesion showed hyperintense signal in both $T_{1}$ and $T_{2}$ weighted images but suppressed in STIR view, characteristic of an intramuscular lipoma (Fig. 3). 


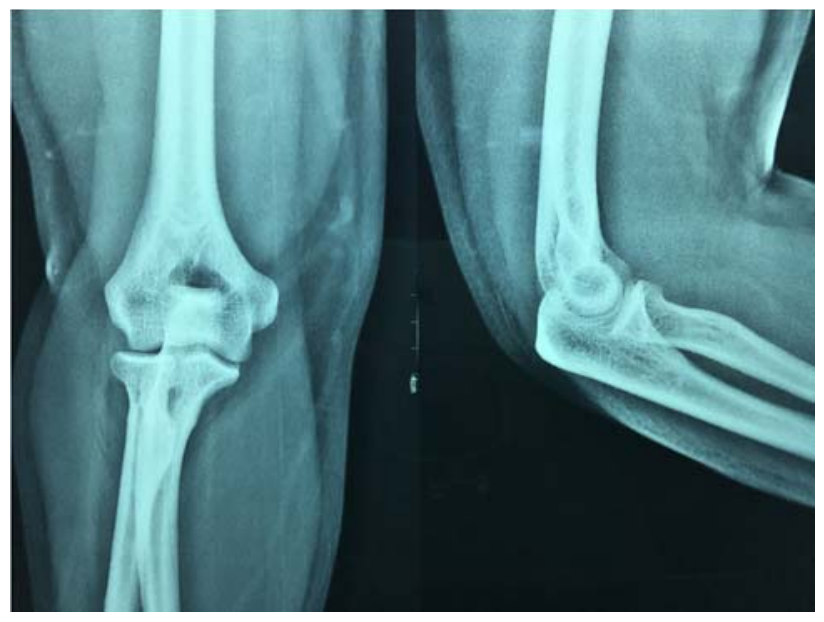

Fig. 2 Plain radiographs of the right elbow (anteroposterior and lateral views), showing no abnormality

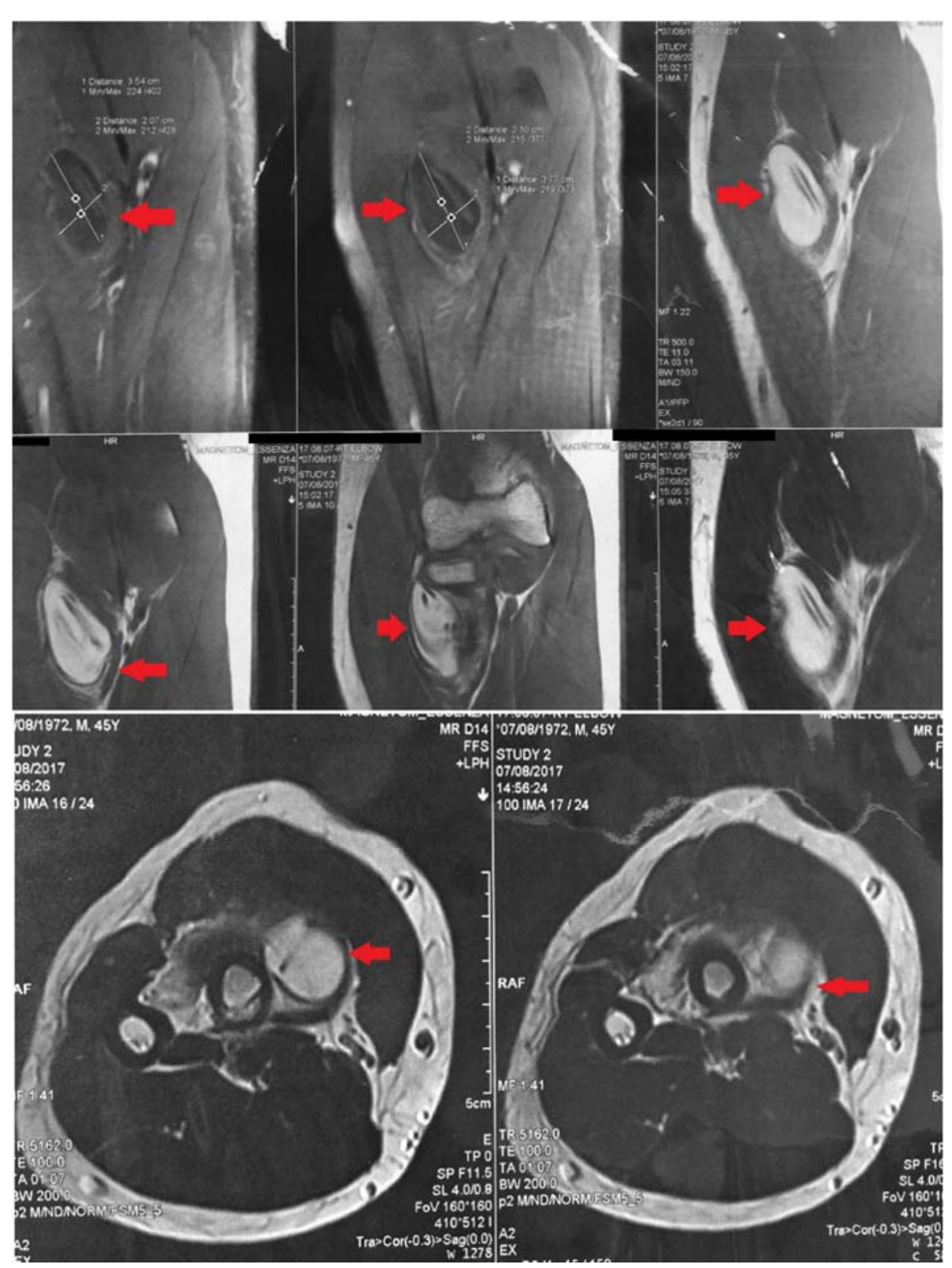

Fig. 3 Magnetic resonance imaging (MRI) of the right proximal forearm region showing soft tissue mass within the supinator muscle (red arrowhead) 


\section{Nerve studies}

Nerve Conduction Velocity (NCV) revealed axonal and demyelinating affection of the PIN, suggesting chronic compression with preservation of superficial radial nerve. Electromyography (EMG) revealed denervation of the muscles innervated by PIN with sparing of radial innervated muscles.

\section{Surgical management}

Under general anesthesia, an excisional biopsy of the lesion was performed with a tourniquet proximal to the arm. Henry's anterior approach to the proximal forearm was used (Fig. 4A). The mobile wad muscles were mobilized laterally, the superficial radial nerve (SRN) below the brachioradialis was intact
(Fig. 4B, C). Supinator muscle was dissected, revealing a fatty encapsulated mass, and the dissection of the mass revealed that it was compressing the PIN with no infiltration (Fig. 4D-I). The nerve was carefully released without damage, and the mass was excised en bloc, with a gross appearance of yellow fatty tissue (Fig. 5A). The mass was sent for histopathological evaluation, which revealed a benign intramuscular lipoma (Fig. 5B). A rehabilitation program was started two weeks post-surgery, after sutures removal. The PIN recovered well three months after surgery and the patient was able to resume his activities. No local recurrence was identified at one-year follow-up.

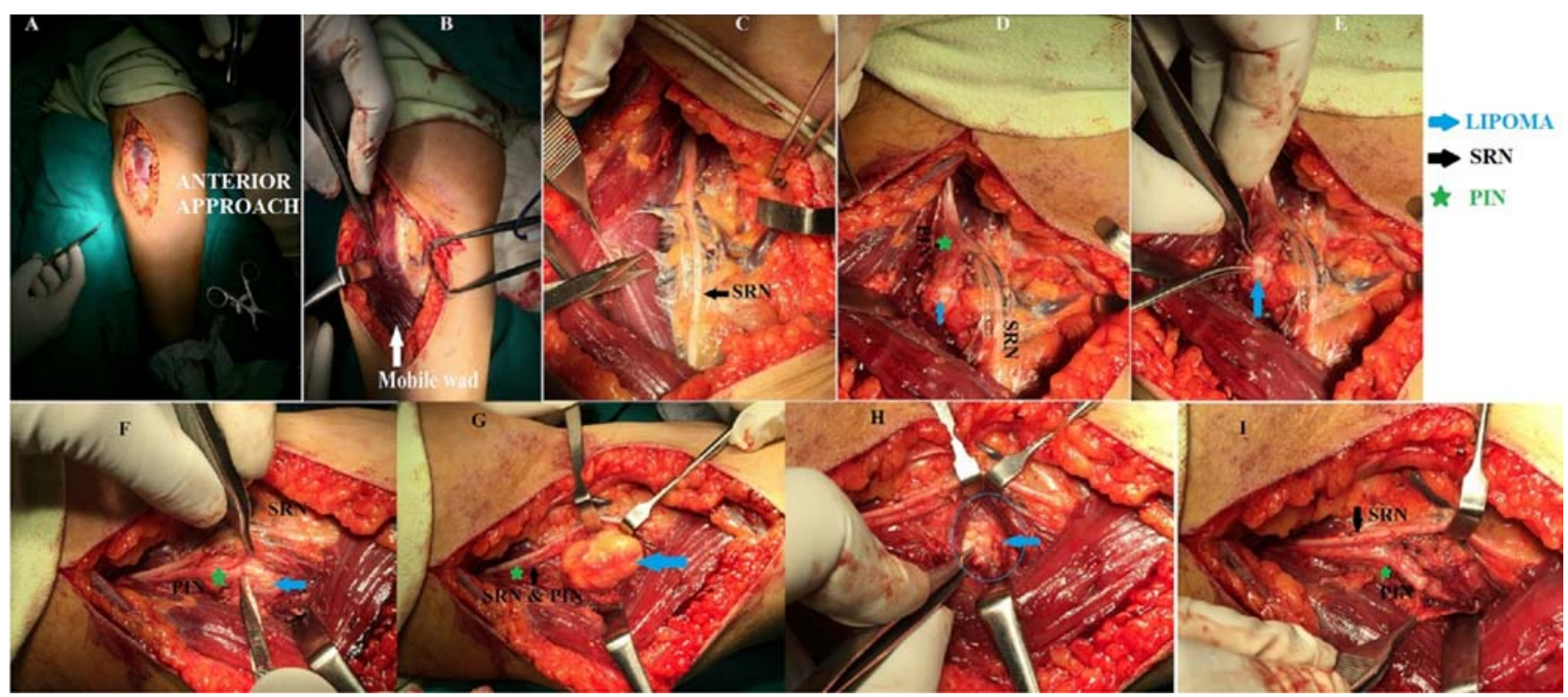

Fig. 4 Surgical steps of the lipoma excision, $\boldsymbol{A}$ : anterior approach to the proximal forearm, $\boldsymbol{B}$ : lateral retraction of the mobile wad muscle group revealing the underlying SRN, C: SRN (black arrowhead) is shown to be stretched over the mass but intact, D: after dissecting the lateral side of the mass (blue arrowhead), the PIN started to appear at the proximal end (green Asterix), E-G: completing dissection of the mass and freeing of the PIN, $\boldsymbol{H}, \mathbf{I}:$ the bed of the mass is shown after en bloc resection with the SRN and PIN freed
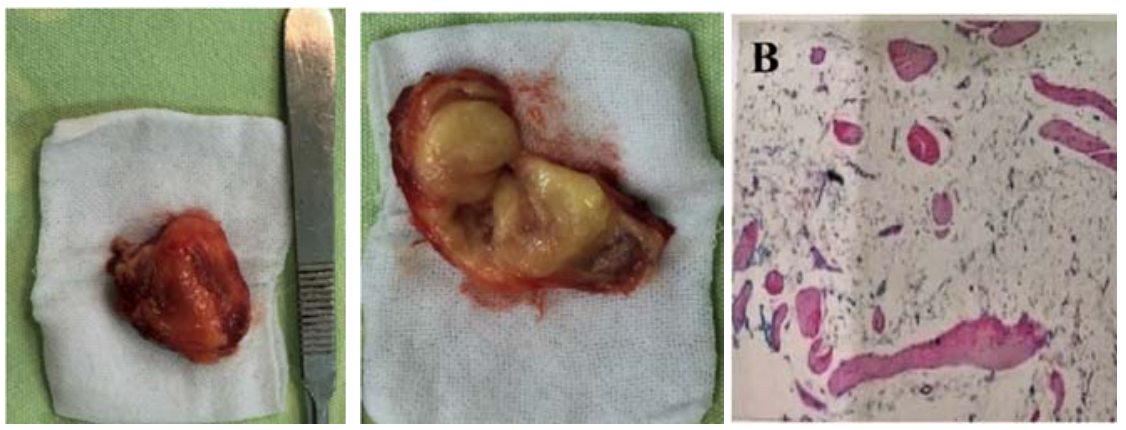

Fig. 5 Pathological appearance of the mass, $\boldsymbol{A}$ : gross picture, $\boldsymbol{B}$ : microscopic picture 


\section{Discussion}

An intramuscular lipoma is considered relatively uncommon, occurring at different anatomical sites, commonly in the large muscles and trunk [6]. It presents grossly as a variable-sized ( $1 \mathrm{~cm}$ up to $25 \mathrm{~cm}$ ), apparently circumscribed mass formed of uniform yellowish adipose tissue [6]. Histologically, it can be divided into the infiltrative, wellcircumscribed, and a mixed type. It presents with a uniform appearance, showing mature univacuolated adipocytes that irregularly infiltrate muscle fibers, the adipocytes' nuclei are small and flattened, showing no nuclear atypia [6].

Compression of the PIN can occur at different anatomical regions, as at the origin of the extensor carpi radialis brevis, at the arcade of Frohse, and the supinator's distal edge muscle $[\mathbf{1}, \mathbf{2}]$. However, entrapment or compression of the PIN can be caused by different benign pathologies such as the parosteal lipoma $[\mathbf{1}, \mathbf{3}, \mathbf{8}]$, intraneural lipoma [4], and a ganglion cyst [5].

The patient with a benign lesion compressing the PIN and leading to nerve entrapment usually presents with an insidious onset of symptoms that depend on the size of the lesion, anatomical location, and its rate of enlargement. Compression of the PIN presents as weakness of fingers extension without sensory affection. If the lesion is large enough to compress the SRN, a sensory loss will be detected $[\mathbf{1}, \mathbf{2}]$. In the present case, the SRN was not affected and the patient presented with partial affection of fingers extension as weakness of the ring and middle finger extension.

As reported by Colasanti et al. [9], a clinical-based diagnosis is of paramount importance to differentiate the PIN entrapment from other pathologies, like cervical spondylosis, causing nerve root compression. However, supplementary imaging investigations should be performed,
Maldonado et al. recommending superficial ultrasound (as a quick and cheap modality) and MRI (imaging of choice) if a lesion was suspected to cause the nerve entrapment that leads to the delay of diagnosis [8].

There is an agreement among surgeons that the recovery of the nerve function depends mainly on the duration of symptoms, and early surgical decompression will offer faster recovery and better functional outcomes $[\mathbf{1}, \mathbf{9}]$. However, despite the success of lesion surgical excision to provide PIN full recovery, in some cases, even with early surgical intervention, full recovery was not gained, requiring further tendon transfer [10]. Vikas et al. suggested that surgical decompression delay beyond 18 months may lead to fibrosis of the muscles innervated by the PIN, increasing the chance for further tendon transfer to gain accepted functional outcomes [1]. The rapid recovery of three months gained in our case was attributed to partial affection of the PIN and early surgical decompression.

The approach used for surgery should offer a wide exposure and a safe window to perform dissection and excision of the mass for the safety of the neural structures. We used the anterior approach for excising the mass, which was recommended in previous studies, as it offered better visualization and access to the lesion, and better use of the anterior approach for excising these lesions $[\mathbf{5}, \mathbf{8}, \mathbf{1 0}]$. However, in their case report, Vikas et al. used the posterior approach to access a parosteal bilobed lipoma, and they reported excellent visualization of PIN and its branches, which guarded against iatrogenic injury nerve during dissecting and excising the lesion [1].

\section{Conclusion}

PIN entrapment secondary to intramuscular lipoma requires early diagnosis and surgical interference to obtain optimum 
functional outcomes. Clinical suspicion of a mass causing nerve entrapment should be confirmed by MRI and neurophysiological studies. Early surgical excision is the treatment of choice in cases with progressive neurological deficit, which can be performed through either anterior or posterior approaches.

\section{Conflict of Interest statement}

Authors state no conflict of interest.

\section{Informed Consent and Human and Animal Rights statement \\ Informed consent has been obtained from all individuals included in this study.}

\section{Authorization for the use of human subjects}

Ethical approval: The research related to human use complies with all the relevant national regulations, institutional policies, is in accordance with the tenets of the Helsinki Declaration.

\section{Acknowledgements}

None.

\section{Sources of Funding}

None.

\section{Disclosures}

None.

\section{References}

1. Vikas V, Bhatia N, Garg J. Posterior interosseous nerve entrapment due to bilobed parosteal lipoma. Journal of Clinical Orthopaedics and Trauma. 2020; 11(Suppl 1):S174-S6.

2. Anania $P$, Fiaschi $P$, Ceraudo $M$, Balestrino $A$, Zaottini $F$, Martinoli $C$ et al. Posterior interosseous nerve entrapments: review of the literature. Is the entrapment distal to the arcade of Frohse a really rare condition? Acta Neurochirurgica. 2018; 16o(9):1857-64.

3. Saaiq $M$, Siddiui S. Posterior Interosseous Nerve Syndrome Resulting from Parosteal Lipoma of the Proximal Radius: An Elusive Diagnosis Yet Excellent
Outcome. World Journal of Plastic Surgery. 2017; 6(1):100-5.

4. Patel AP, Aoun SG, Al Tamimi M. Intraneural Posterior Interosseous Nerve Lipoma with Complete Paralysis: Case Report and Review of the Literature. Cureus. 2018; 10(5):e2689.

5. Purohit S, Prabhakar A, Raj A, Marathe N, Shah S. A rare case of posterior interosseous nerve palsy due to ganglion cyst arising from supinator muscle. Journal of Clinical Orthopaedics and Trauma. 2020; 11(4):665-7.

6. McTighe $S$, Chernev I. Intramuscular lipoma: a review of the literature. Orthop Rev (Pavia). 2014; 6(4):5618.

7. Charifa A, Azmat CE, Badri T. Lipoma Pathology. 2020, StatPearls, Treasure Island (FL), StatPearls Publishing.

8. Maldonado AA, Howe BM, Spinner RJ. Posterior interosseous nerve discontinuity due to compression by lipoma: report of 2 cases. Journal of Neurosurgery. 2017; 126(5):1698-701.

9. Colasanti $R$, lacoangeli $M$, Di Rienzo $A$, Dobran $M, D i$ Somma $L$, Nocchi $N$ et al. Delayed diagnosed intermuscular lipoma causing a posterior interosseous nerve palsy in a patient with cervical spondylosis: the "priceless" value of the clinical examination in the technological era. II Giornale di chirurgia. 2016; 37(1):42-5.

10. Unal M, Kose O, Guler F, Egerci OF. Posterior interosseous nerve palsy caused by a parosteal lipoma seated over the proximal radius; a case report and review of the literature. Hand Microsurg. 2018; 7:149-59. 